\title{
多色量子点表面等离子体耦合苂光发射性质
}

\author{
刘晓庆刘倩谢凯信曹炼晖蔡伟鹏李耀群*
}

(厦门大学化学化工学院化学系与现代分析科学重点实验室 厦门 361005)

\begin{abstract}
摘要 以統基小分子为配体水相合成 $\mathrm{CdTe}$ 量子点, 通过调节回流时间调控其粒径大小. 由于量子点的宽谱激发特性, 在蓝光(473 nm)或绿光(532 nm)条件下, 纳米金属薄膜表面不同发射波长的 $\mathrm{CdTe}$ 量子点均可被激发而与金属表面等离 子体发生耦合相互作用, 从而在棱镜一侧发出高度定向的偏振荧光, 其苂光特性与样品厚度密切相关. 表面等离子体 耦合荧光发射法(SPCE)具有波长分辨性质，不同颜色的量子点在不同角度定向发射，发射波长越长，角度越小７20 $\mathrm{nm}$ 和 $630 \mathrm{~nm}$ 量子点的自由空间发射荧光光谱呈现交叠, 然而, 基于 SPCE 的波长分辨性质, 我们通过改变检测角度避 开光谱重叠, 在棱镜一侧的 $43^{\circ}$ 和 $51^{\circ}$ 处分别得到了两种量子点的 SPCE 荧光单峰. 量子点是 SPCE 在多通路、高通量 检测应用中荧光物种的理想选择.
\end{abstract}

关键词 表面等离子体耦合苂光发射; $\mathrm{CdTe}$ 量子点; 宽谱激发; 波长连续可调; 波长分辨

\section{Surface Plasmon-coupled Emission of Multicolor Quantum Dots}

\author{
Liu, Xiaoqing Liu, Qian Xie, Kaixin Cao, Shuohui Cai, Weipeng Li, Yaoqun* \\ (Department of Chemistry and Key Laboratory of Analytical Sciences, College of Chemistry and Chemical Engineering, \\ Xiamen University, Xiamen 361005)
}

\begin{abstract}
Aqueous CdTe quantum dots (QDs) were synthesized employing thiols as stabilizing ligands and their emission wavelengths could be tuned by controlling the reflux time. Surface plasmon-coupled emission (SPCE) properties of multicolor CdTe QDs were studied by using a laboratory-built multifunctional spectrofluorimeter. To fabricate a CdTe QDs-doped complete film onto the surface of continuous thin metal films, CdTe QDs were spin-coated at $3000 \mathrm{r} / \mathrm{min}$ with poly(vinyl alcohol) (PVA) solution of various concentrations. For SPCE measurements, the spin-coated slides were attached to a semi-cylindrical prism made of fused silica with a refractive-index-matching fluid and then positioned on a precise rotary stage that allowed for excitation and emission observation at any angle relative to the vertical axis of the stage. For excitation, we used the reverse Kretschmann (RK) configuration and the incident light was normal to the sample interface. The spectra were measured using a monochromator equipped with a photomultiplier tube. The experimental results showed CdTe QDs located nearby continuous thin metal film could be excited using either blue $(473 \mathrm{~nm})$ or green $(532 \mathrm{~nm})$ laser as a result of their broad excitations and their emissions resulting from the surface plasmon coupling were strongly directional, highly polarized and closely related to sample thickness. Because of the wavelength-resolution property of SPCE, CdTe QDs with different emission wavelengths were found to emit at different fixed angles, longer wavelengths corresponding to smaller angles. The free space emission spectra of $720 \mathrm{~nm}$ and $630 \mathrm{~nm}$ CdTe QDs in a mixture were overlapped and could not be separated at any detection angle. However, owing to the unique wavelength resolution ability of SPCE, the spectra of $720 \mathrm{~nm}$ and $630 \mathrm{~nm} \mathrm{CdTe}$ QDs were obtained at $43^{\circ}$ and $51^{\circ}$ on the prism side, respectively. The determination of each component of the mixtures of CdTe QDs was achieved by changing the detection angle to avoid spectral overlap. Therefore, QDs are ideal fluorophores for SPCE in high-throughput, multiplex analysis application.

Keywords surface plasmon-coupled emission (SPCE); CdTe quantum dots; broad excitation; continuous tunable emissions; wavelength resolution
\end{abstract}

\section{1 引言}

表面等离子体耦合定向荧光法(Surface plasmoncoupled emission, SPCE $)^{[1 \sim 3]}$ 可以看作表面等离子体共振 (Surface plasmon resonance, SPR)的逆过程, 它是指位于 纳米金属近场作用范围内的激发态苂光团与金属表面
等离子体相互作用, 从而在棱镜侧产生不同于各向同性 自由发射的高度定向的偏振辐射, 进而提高荧光信号收 集效率的一种新型表面增强荧光方法. 与传统意义上的 荧光相比, SPCE 具有高度定向、高度偏振、距离依赖及 波长分辨等诸多独特性质，在提高方法灵敏度、背景抑 制及多通路、高通量检测方面独具优势，在分析检测研

* E-mail: yqlig@xmu.edu.cn

Received July 16, 2012; published September 9, 2012.

Project supported by the National Natural Science Foundation of China (Nos. 21127005, 20975084), the 973 Program of China (No. 2013 CB933703), Ph.D. Programs Foundation of Ministry of Education of China (No. 20110121110011).

项目受国家自然科学基金(Nos. 21127005, 20975084)、国家 973 计划(No. 2013CB933703)和教育部博士点基金(No. 20110121110011)资助. 
究领域受到越来越多的关注 ${ }^{[4 \sim 7]}$, 我们利用距离效应分 别建立了核酸和蛋白质的 SPCE 检测方案 ${ }^{[8,9]}$.

传统有机荧光染料探针在 SPCE 中的应用常常面临 着如下问题: (1) SPCE 通常使用激光器等强光作为光源, 一般说来, 有机苂光染料光稳定性差, 易漂白; (2)有机 荧光染料为窄带激发, 为了满足不同的检测需求, 通常 需要配备不同波段的激发光源, 而激光光源通常较为昂 贵, 不利于仪器的推广使用; (3)不同发射波长的有机苂 光染料通常其激发波长不尽相同, 在同一体系中很难实 现同一激发波长下的多组分同时标记. 然而, 作为一种 特殊的纳米苂光材料, 量子点具有光稳定性好、激发光 谱宽、发射光谱连续可调以及荧光量子产率高等许多优

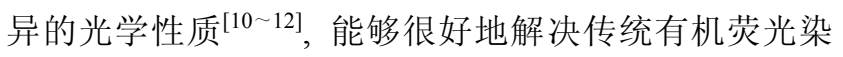
料分子在 SPCE 应用中面临的问题, 是 SPCE 中苂光物 种的理想选择 ${ }^{[6,13]}$.

近年来，随着量子点的合成技术尤其是水相合成技 术的不断发展成熟, 其在化学生物传感、免疫反应及生 物成像等诸多领域发挥着重要作用 ${ }^{[14 ~ 16]}$. 本工作以颈 基小分子为配体直接水相合成了不同粒径 $\mathrm{CdTe}$ 量子点, 系统考察了光滑金属薄膜表面 CdTe 量子点-PVA 样品 膜的 SPCE 性质以及其一元激发多元发射特性在 SPCE 应用中的优越性.

\section{2 结果与讨论}

\subsection{CdTe 量子点的 SPCE 性质}

量子点具有量子尺寸效应，表现为粒径越大，苂光 发射波长越长, 在水相合成中可以通过调节回流时间控 制量子点的粒径进而调控其最佳荧光发射波长. 图 1 插 图中量子点发光颜色随回流时间的增长由无色过渡到 绿色最后变为红色, 直观地反映了量子点的这一量子尺 寸效应. 我们以不同的颈基小分子为配体合成了波长在 $520 \sim 720 \mathrm{~nm}$ 的 CdTe 量子点. 其中, 荧光发射波长较长

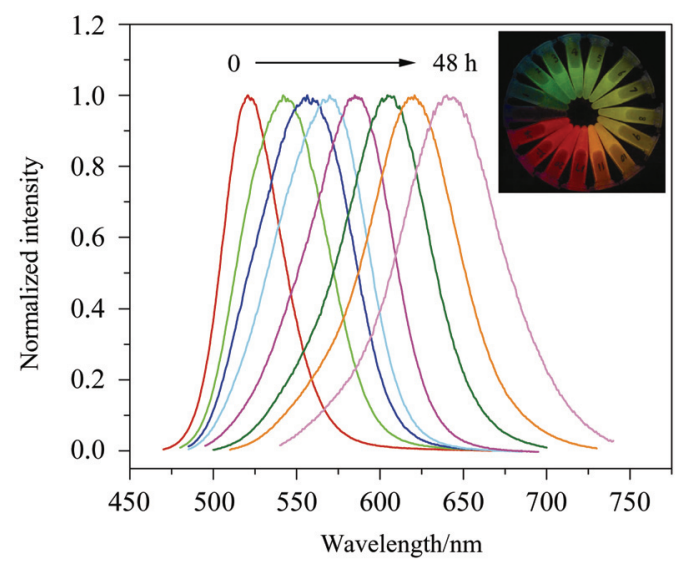

图 1 不同回流时间 $\mathrm{CdTe}$ 量子点的苂光发射光谱

Figure 1 Fluorescence spectra of CdTe QDs with different reflux time From left to right, 1, 3, 5, 7, 10, 17, 24, $48 \mathrm{~h}$. The inset shows photograph of CdTe QDs with different reflux time under $365 \mathrm{~nm}$ UV irradiation $(\geqslant 700 \mathrm{~nm})$ 的量子点是以颈基丙酸为配体获得的. 扫描 不同回流时间以旒基乙酸为配体的 CdTe 量子点的荧光 光谱, 如图 1 所示, 在 $0 \sim 48 \mathrm{~h}$ 内, 颈基乙酸稳定的 CdTe 量子点波长在 $520 \sim 640 \mathrm{~nm}$ 范围内连续变化.

传统意义上的苂光是沿自由空间发射的各向同性 的苂光信号，当将荧光物质置于粗糙或超薄光滑金属表 面，二者之间复杂的相互作用使得其光学性质如荧光强 度、苂光量子产率及空间分布等受到极大的影响 ${ }^{[17,18]}$. 金属表面与苂光团之间主要存在荧光猝灭作用、表面等 离子体耦合作用及空间自由发射作用. 当二者距离小于 $10 \mathrm{~nm}$ 时, 最有可能发生荧光猝灭过程, 而当距离超过 $500 \mathrm{~nm}$ 时, 则是自由空间发射(Free Space, FS)占主导. 而对于 SPCE 来说, 其距离要控制在猝灭区域以外而又 尽量靠近金属表面，这样可以使得荧光团的偶极子能够 更好地与金属等离子体发生相互作用. 由于光滑纳米金 属薄膜表面等离子体的作用，苂光团的菼光发射表现出 高度定向、高度偏振及波长分辨等区别于 FS 的独特的 光学性质 ${ }^{[1 \sim 6]}$.

图 2 为 $50 \mathrm{~nm} \mathrm{Au}$ 膜表面上 $\mathrm{CdTe}$ 量子点-PVA 样品 膜(膜厚 $30 \mathrm{~nm}, \lambda=590 \mathrm{~nm}$ ) 的苂光强度随检测角度变化 的极坐标图, 由图可知, $\mathrm{Au}$ 膜表面 $\mathrm{CdTe}$ 量子点的 SPCE 信号表现出高度定向性质, 检测角度越接近 $\pm 51^{\circ}$, 苂 光强度越强, 采集到的荧光图像越亮, 即 $\mathrm{Au}$ 膜的存在 使得量子点的发光性质发生改变, SPCE 信号在 $\pm 51^{\circ}$ 处 呈现高度定向发射.

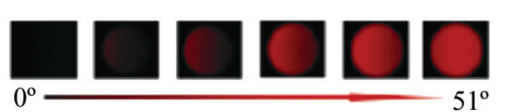

90

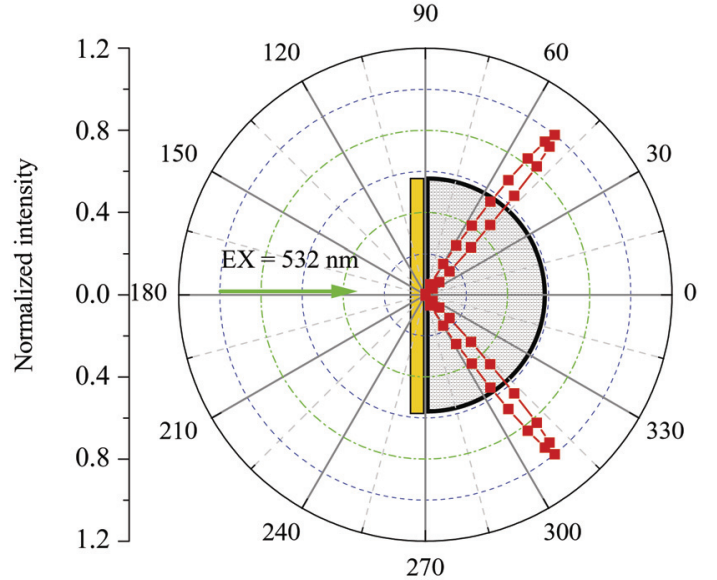

图 $250 \mathrm{~nm} \mathrm{Au}$ 膜表面 CdTe 量子点-PVA 样品膜苂光信号随着角度 变化分布极坐标图(插图为不同检测角拍摄的 SPCE 信号图)

Figure 2 Angular distributions of SPCE intensity for QDs-PVA film on a 50-nm-thick Au film (The inset is the photograph of SPCE signals at different detection angles)

荧光团与金属表面等离子体耦合后产生的 SPCE 信 号具有偏振性, 在发射光路放置偏振片, 考察了 $\mathrm{CdTe}$ 量子点-PVA 样品的荧光信号偏振性. 在最佳检测角 $\left(51^{\circ}\right)$ 测得的 SPCE 为完全 $\mathrm{p}$-偏振的信号 (图 3A), 在自由 
空间发射荧光信号则不表现出这一独特的偏振性质(图 3B).
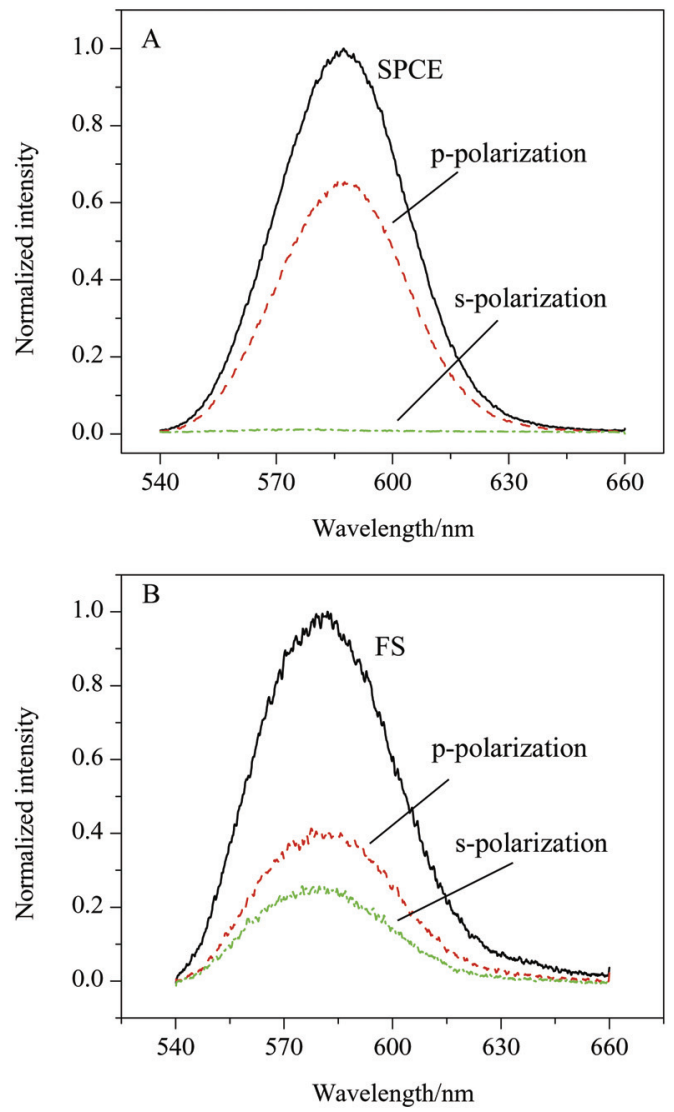

图 3 CdTe 量子点-PVA 样品膜不同偏振发射的 SPCE 光谱图(A)和 FS (B) 苂光光谱图

Figure 3 SPCE (A) and FS (B) spectra for CdTe QDs-PVA film with pand s-polarizations

量子点的 SPCE 信号具有波长分辨性, 在不同检测 角度下发射光谱的峰位置不同. 由图 4A 可知, 随着检 测角度的变化, SPCE 苂光光谱发生位移, 检测角度越大, 最大发射波长越小; 而 FS 苂光光谱则不会随检测角度 发生位移(图 4B 所示). 该光谱位移来自于 SPCE 自身的 角度色散特性，而该色散性则源于纳米金属薄膜介电常 数的波长相关性 ${ }^{[19]}$.

\section{2 不同厚度 $\mathrm{CdTe}$ 量子点样品膜的 SPCE 性质}

配制一系列 $\mathrm{CdTe}$ 量子点的 PVA 溶液, 其中 PVA 质量分数分别为 $1 \%, 2 \%, 4 \%$ 和 $6 \%$, 在金膜基底上 $(2 \mathrm{~nm}$ $\mathrm{Cr} / 50 \mathrm{~nm} \mathrm{Au} / 5 \mathrm{~nm} \mathrm{SiO})_{2}$ ) 以旋转涂布成膜的方式制备样 品膜, 样品膜厚度通过 PVA 浓度来控制, 浓度越高, 样 品膜越厚. 图 5 为不同厚度量子点的 SPCE 角度定向性 和偏振性. $1 \% \mathrm{PVA}$ 量子点 SPCE 苂光信号定向分布在 $54^{\circ}$, 呈 $\mathrm{p}$-偏振性质; 随着样品膜厚度的增加, 即 PVA 浓 度分别为 $2 \%$ 和 $4 \%$, 量子点定向角变化的同时发生偏振 翻转, 为完全 $\mathrm{s}$-偏振的光; 而当 PVA 浓度继续增加至 $6 \%$ 时, SPCE 在 $43^{\circ}, 54^{\circ}, 67^{\circ}$ 三个角度定向发射, 分别表
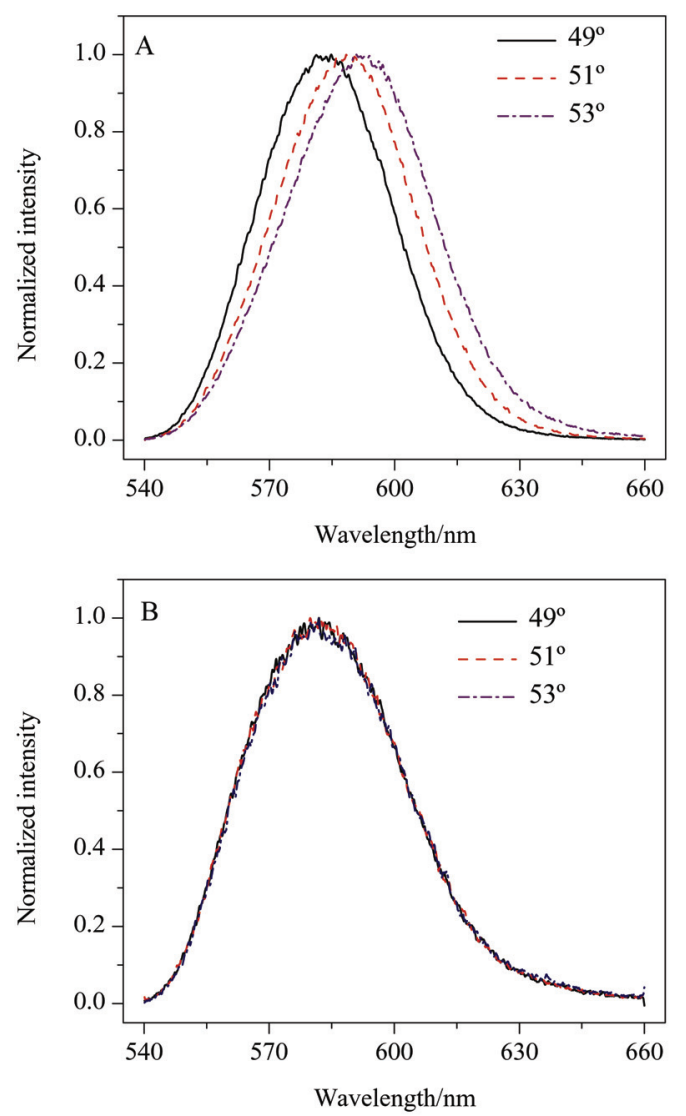

图 4 不同检测角度下 CdTe 量子点-PVA 样品膜的 SPCE (A) 和 FS (B) 苂光光谱图

Figure 4 SPCE (A) and FS (B) spectra for CdTe QDs-PVA film at different detection angles

现出 $\mathrm{s}, \mathrm{p}, \mathrm{s}$ 偏振性质，且在 $54^{\circ}$ 处的信号强度最强，可见， 量子点的 SPCE 性质与样品厚度息息相关. 我们可以通 过 SPCE 定向角的位置利用 Winspall3.01 软件 ${ }^{[20,21]}$ 模拟 计算各样品厚度，得量子点的 $1 \%, 2 \%, 4 \%$ 和 $6 \%$ PVA 膜 的厚度分别为 $28,90,130$ 和 $352 \mathrm{~nm}$.

\section{3 不同激发波长下 CdTe 量子点的 SPCE 性质}

分别以波长 $532 \mathrm{~nm}$ 和 $473 \mathrm{~nm}$ 的光源激发 $\mathrm{Au}$ 膜表 面 CdTe 量子点-PVA样品膜 $(\lambda=590 \mathrm{~nm})$, 均在棱镜一侧 特定角度 $\left(51^{\circ}\right)$ 收集到高度定向的偏振荧光信号, 且苂光 信号强度随角度变化趋势基本一致(图 6). 可见, 鉴于其 宽谱带激发特性, 在较大的波长范围内量子点均能被激 发而与金属表面等离子体发生耦合相互作用，因而可以 选择相对便宜的较长波长激发光源, 在 SPCE 光源选择 方面表现出无可比拟的优越性.

\section{4 不同发射波长 CdTe 量子点的 SPCE 性质}

实验考察了不同发射波长的 $30 \mathrm{~nm}$ 厚 $\mathrm{CdTe}$ 量子点PVA 膜的 SPCE 荧光特性. Au 膜表面不同发射波长的 $\mathrm{CdTe}$ 量子点均能被同一光源激发, 并在棱镜一侧发射 高度定向、完全 $\mathrm{p}$-偏振的 SPCE 信号，且不同发射波长 量子点的定向角不同. 如图 7A 所示, 实验测得 540, 

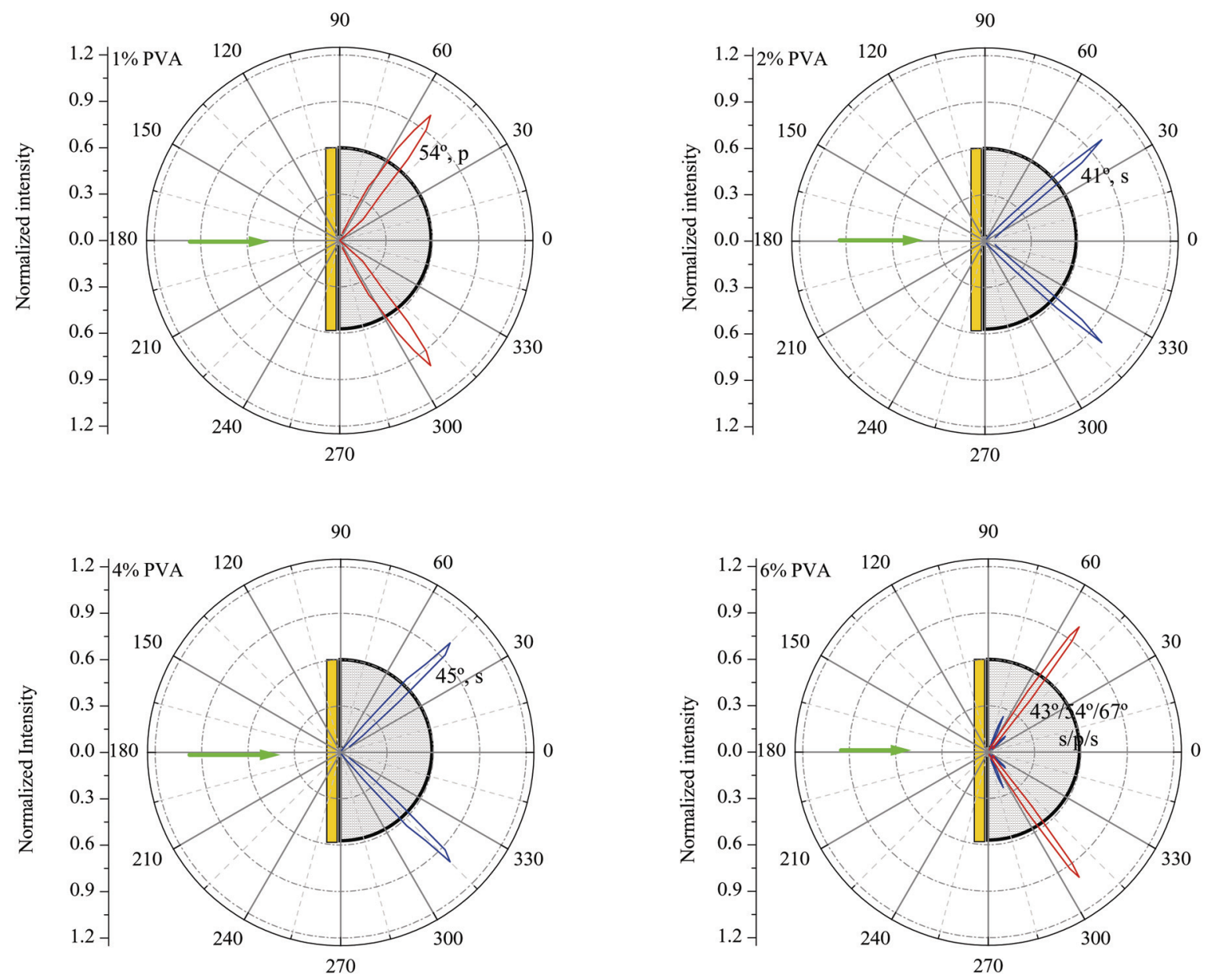

图 5 不同厚度 $\mathrm{CdTe}$ 量子点-PVA 样品膜苂光信号强度随着角度变化分布极坐标图

Figure 5 Angular distributions of SPCE intensity for QDs-PVA films with different thicknesses

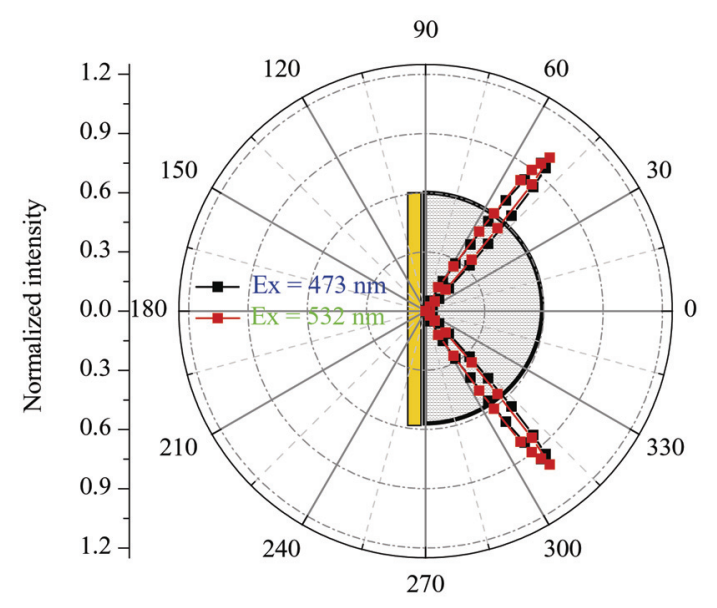

图 $6 \mathrm{CdTe}$ 量子点-PVA 样品膜在 $473 \mathrm{~nm}$ (黑线)和 $532 \mathrm{~nm}$ (红线)激发 下 SPCE 随着角度变化分布极坐标图

Figure 6 Angular distributions of SPCE intensity for QDs-PVA film with $473 \mathrm{~nm}$ (black dotted line) and $532 \mathrm{~nm}$ (red dotted line) excitation, respectively

570, 590, 630 和 $720 \mathrm{~nm}$ CdTe 量子点的 SPCE 定向角分 别为 $61^{\circ}, 57^{\circ}, 51^{\circ}, 48^{\circ}$ 和 $45^{\circ}$, 波长越长, 角度越小. 用
Winspall3.01 软件模拟相应条件下的 SPR 反射曲线, 如 图 7B 所示, 波长为 $540 \mathrm{~nm}$ 时, 反射率曲线分布在较宽 的一个角度范围内，表面等离子体共振角约为 $61^{\circ}$, 随 着发射峰位置的红移, 峰形逐渐变得尖锐, 且表面等离 子体共振角越来越小, 变为 $47^{\circ}$, 与 SPCE 实验测量结果 基本一致. 可见，在同一激发光源下，不同粒径的 $\mathrm{CdTe}$ 量子点均能被激发并与金属薄膜表面的表面等离子体 发生耦合相互作用, 且由于 SPCE 的波长分辨作用其定 向角度不同.

有机苂光染料由于窄带激发的限制很难实现同一 体系同一激发波长下的多组分同时标记，量子点的一元 激发多元发射性质使得其成为多组分标记同时检测中 荧光物种的理想选择. 我们将最佳发射波长为 630 和 $720 \mathrm{~nm}$ 的 CdTe 量子点按一定比例混合后在 $50 \mathrm{~nm} \mathrm{Ag}$ 膜表面成膜, 考察其苂光性质, 如图 8 所示, 两种 $\mathrm{CdTe}$ 量子点在 FS 方向荧光发射光谱部分重叠, 改变检测角 度并不能改善分辨程度，在实际检测应用中不利于多组 分同时标记的分别检测. 然而, SPCE 具有波长分辨性质, 在不同检测角度可以得到不同的苂光发射光谱，鉴 

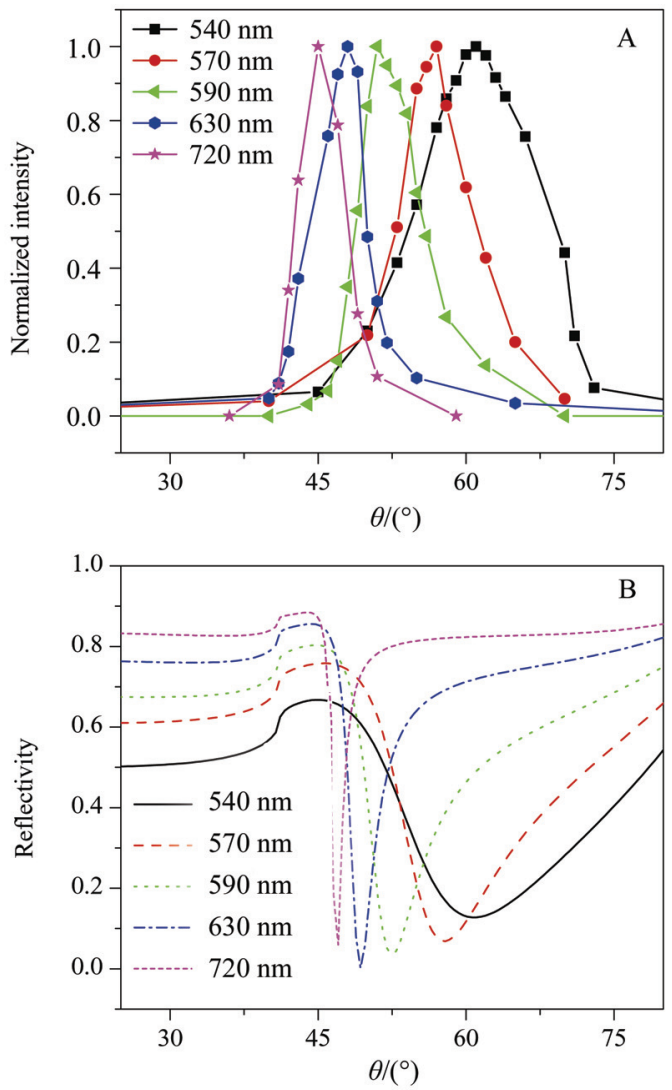

图 7 不同发射波长 CdTe 量子点-PVA 样品膜的 SPCE 信号分布曲线 (A)及 SPR 反射模拟曲线(B)

Figure 7 SPCE intensity distribution curves (A) and simulated SPR curves (B) of QDs-PVA with different emission wavelengths

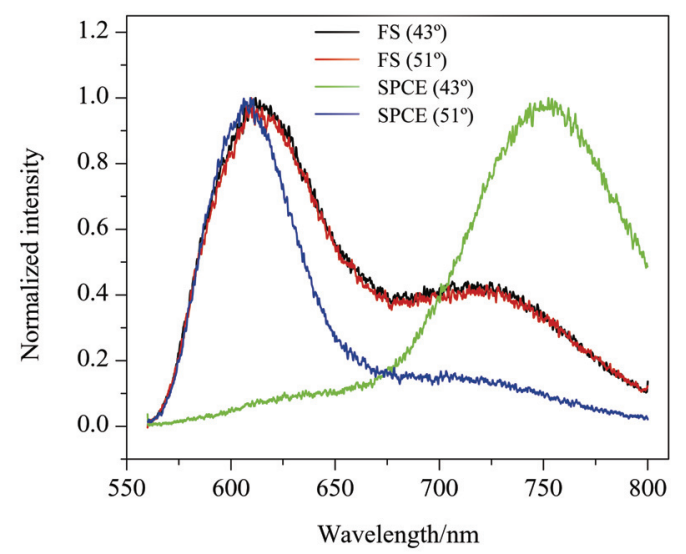

图 $8 \mathrm{CdTe}$ 量子点混合物-PVA 样品在不同检测角度下的 FS 光谱及 SPCE 光谱

Figure 8 FS and SPCE spectra of the mixture of CdTe QDs-PVA at different detection angles

于不同发射波长量子点在 SPCE 方向的定向发射角不同, 我们通过改变检测角度避开光谱重叠, 在 $43^{\circ}$ 和 $51^{\circ}$ 处分 别得到了 720 和 $630 \mathrm{~nm}$ 量子点的荧光单峰, 两种量子点 的 SPCE 信号可以通过检测角度的变化而被同时分别检 测, 因而将波长连续可调的量子点与 SPCE 波长分辨性
质相结合在多通道、高通量检测中具有广阔应用前景.

\section{3 结论}

由于与纳米金属薄膜表面等离子体发生耦合作用, $\mathrm{CdTe}$ 量子点的苂光发射表现出定向性、偏振性及波长 分辨性等独特性质, 且与样品厚度密切相关. 鉴于量子 点的 “一元激发多元发射” 特性及 SPCE 的波长分辨性 质，金属薄膜表面不同发射波长的量子点可被同一光源 激发而在不同角度定向发射, 波长越长, 角度越小. 研 究结果表明, 量子点是 SPCE 中荧光物种的理想选择, 可望构成多色探针，应用于同一体系中同一激发波长下 多组分分析物的多通道、高通量检测; 和常规苂光法相 比, SPCE 提供独特的波长分辨能力.

\section{4 实验部分}

\section{1 量子点的合成}

CdTe 量子点的水相合成参照文献[22 24]稍作改 进, 分别以颈基乙酸(Thioglycolic acid, TGA)、颈基丙酸 (Mercaptopropionic acid, MPA)和颈基乙胺(Cysteinamine, $\mathrm{CA}$ )为配体, $\mathrm{Te}$ 粉为碲源, 粒径大小通过回流时间调控.

\section{2 样品制备}

在厦门大学微机电中心采用磁控溅射法, 于石英载 玻片上依次浌射 $2 \mathrm{~nm} \mathrm{Cr}$ 和 $50 \mathrm{~nm} \mathrm{Au}$ ，制得金纳米薄膜; 于石英载玻片上依次溅射 $2 \mathrm{~nm} \mathrm{Cr}, 50 \mathrm{~nm} \mathrm{Ag}$, 制得银纳 米薄膜. 其中, $2 \mathrm{~nm} \mathrm{Cr}$ 层用于增强金属薄膜在石英玻璃 上的粘附性.

配制 CdTe 量子点的 PVA 溶液, 通过旋转涂布成膜 方式在金属薄膜表面制备 CdTe 量子点-PVA 样品膜, 匀 胶机参数设置为 $3000 \mathrm{r} / \mathrm{min}$ 及 $30 \mathrm{~s}$, 样品厚度由 PVA 浓 度控制.

\section{3 光谱测定}

使用实验室自主搭建的多功能荧光光谱仪考察 $\mathrm{CdTe}$ 量子点的 SPCE 性质, 图 9 为 Reverse Kretschmann (RK)模式下的 SPCE 检测装置示意图.

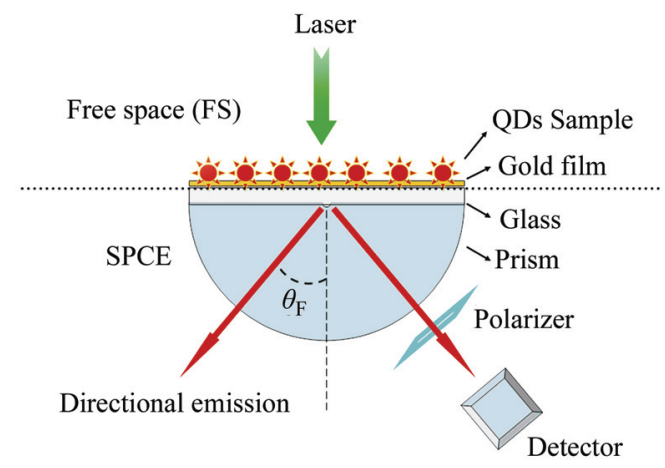

图 9 Reverse Kretschmann (RK) 模式下 SPCE 苂光检测装置示意图 Figure 9 Geometry for SPCE measurement with the Reverse Kretschmann (RK) configurations 
量子点通过 PVA 旋转涂布成膜方式依附于纳米级 光滑金属薄膜表面，负载量子点传感膜的石英玻璃片通 过光学常数匹配的溶液(如甘油)粘附在半圆柱型棱镜上, 并通过自制样品架固定在精密旋转台上. 光源垂直照射 金属膜激发量子点样品, 在金属膜前端收集其沿自由空 间发射的各向同性的菼光信号，即 FS 信号，而在金属 膜后端即棱镜一侧收集高度定向的表面等离子体耦合 荧光发射信号, 即 SPCE 信号. SPCE 具有波长分辨性质, 不同发射波长的量子点的 SPCE 定向角各不相同. 然而, 由于激发光路可以随精密旋转台实现 $360^{\circ}$ 旋转, 我们可 以调节精密旋转台的位置使量子点传感膜不同角度发 射的菼光信号以一定角度选择性地通过单色仪进入检 测器, 实现苂光信号的收集. 苂光信号的偏振性质考察 是通过在发射光路放置不同方向的偏振片实现的.

\section{References}

[1] Lakowicz, J. R. Anal. Biochem. 2004, 324, 153.

[2] Geddes, C. D.; Gryczynski, I.; Malicka, J.; Gryczynski, Z.; Lakowicz, J. R. J. Fluoresc. 2004, 14, 119.

[3] Malicka, J.; Gryczynski, I.; Gryczynski, Z.; Lakowicz, J. R. Anal. Chem. 2003, 75, 6629 .

[4] Chowdhury, M. H.; Malyn, S. N.; Aslan, K.; Lakowicz, J. R.; Geddes, C. D. J. Phys. Chem. B 2006, 110, 22644.

[5] Yuk, J. S.; Michal, T.; McDonagh, C.; MacCraith, B. D. Biosens. Bioelectron. 2010, 25, 1344.

[6] Jin, L.-H.; Li, S.-M.; Cho, Y.-H. Biosens. Bioelectron. 2012, 33, 284.

[7] Cao, S.-H.; Xie, T.-T.; Cai, W.-P.; Li, Y.-Q. Chem. J. Chin. Univ 2010, 31，61. (曹炼晖，谢堂堂，蔡伟鹏，李耀群，高等学校化学
学报, 2010,31, 61.)

[8] Cao, S.-H.; Xie, T.-T.; Cai, W.-P.; Liu, Q.; Li, Y.-Q. J. Am. Chem. Soc. 2011, 133, 1787.

[9] Xie, T. T.; Liu, Q.; Cai, W. P.; Chen, Z.; Li, Y. Q. Chem. Commun. 2009, (22), 3190.

[10] Gaponik, N.; Rogach, A. L. Phys. Chem. Chem. Phys. 2010, 12 , 8685 .

[11] Li, Z.; Zhu, X.; Dong, C. Q.; Huang, X. Y.; Chen, H. J.; Ren, J. C. Chem. J. Chin. Univ. 2010, 31, 1905. (李众, 祝欣, 董朝青, 黄香 宜，陈虹锦，任吉存，高等学校化学学报, 2010, 31, 1905.)

[12] Resch-Genger, U.; Grabolle, M.; Cavaliere-Jaricot, S.; Nitschke, R.; Nann, T. Nat. Methods 2008, 5, 763 .

[13] Gryczynski, I.; Malicka, J.; Jiang, W.; Fischer, H.; Chan, W. C. W.; Gryczynski, Z.; Grudzinski, W.; Lakowicz, J. R. J. Phys. Chem. B 2005, 109, 1088 .

[14] de Aberasturi, D. J.; Montenegro, J.-M.; de Larramendi, I. R.; Rojo, T.; Klar, T. A.; Alvarez-Puebla, R.; Liz-Marzán, L. M.; Parak, W. J. Chem. Mater. 2012, 24, 738.

[15] Mattoussi, H.; Palui, G.; Na, H. B. Adv. Drug Delivery Rev. 2012, $64,138$.

[16] Michalet, X.; Pinaud, F. F.; Bentolila, L. A.; Tsay, J. M.; Doose, S.; Li, J. J.; Sundaresan, G.; Wu, A. M.; Gambhir, S. S.; Weiss, S. Science 2005, 307, 538 .

[17] Hellen, E. H.; Axelrod, D. J. Opt. Soc. Am. B 1987, 4, 337.

[18] Lakowicz, J. R. Anal. Biochem. 2005, 337(2), 171.

[19] Matveeva, E.; Malicka, J.; Gryczynski, I.; Gryczynski, Z.; Lakowicz, J. R. Biochem. Biophys. Res. Commun. 2004, 313, 721.

[20] Salamon, Z.; Macleod, H. A.; Tollin, G. Biochim. Biophys. Acta 1997, 1331, 117.

[21] Salamon, Z.; Macleod, H. A.; Tollin, G. Biochim. Biophys. Acta 1997, 1331, 131 .

[22] Liang, J. R.; Zhong, W. Y.; Yu, J. S. Chem. J. Chin. Univ. 2009, 30, 14. (梁佳然, 钟文英, 于俊生, 高等学校化学学报, 2009, 30, 14.)

[23] Kuang, R.; Kuang, X.; Pan, S.; Zheng, X.; Duan, J.; Duan, Y. Microchim. Acta 2010, 169, 109.

[24] Zou, L.; Gu, Z.; Zhang, N.; Zhang, Y.; Fang, Z.; Zhu, W.; Zhong, X J. Mater. Chem. 2008, 18, 2807. 